OPEN ACCESS

Edited by:

Samiul Hasan,

University of Central Florida,

United States

Reviewed by:

Yuan Liao,

Chalmers University of

Technology, Sweden

Takahiro Yabe,

Purdue University, United States

*Correspondence:

Xinyu Gao

xy.gao@tamu.edu

${ }^{\dagger}$ These authors have contributed equally to this work

Specialty section:

This article was submitted to Transportation and Transit Systems, a section of the journal

Frontiers in Built Environment

Received: 18 September 2020

Accepted: 29 December 2020

Published: 04 February 2021

Citation:

Gao X, Fan C, Yang Y, Lee S, Li Q

Maron M and Mostafavi A (2021) Early Indicators of Human Activity During COVID-19 Period Using Digital Trace

Data of Population Activities.

Front. Built Environ. 6:607961.

doi: 10.3389/fbuil.2020.607961

\section{Early Indicators of Human Activity During COVID-19 Period Using Digital Trace Data of Population Activities}

\author{
Xinyu Gao ${ }^{1 * t}$, Chao Fan ${ }^{1 \dagger}$, Yang Yang ${ }^{2}$, Sanghyeon Lee ${ }^{3}$, Qingchun $\mathrm{Li}^{1}$, Mikel Maron ${ }^{4}$ and \\ Ali Mostafavi ${ }^{1}$
}

${ }^{1}$ Department of Civil and Environmental Engineering, Texas A\&M University, College Station, TX, United States, ${ }^{2}$ Department of Computer Science and Engineering, Texas A\&M University, College Station, TX, United States, ${ }^{3}$ Department of Electrical and Computer Engineering, Texas A\&M University, College Station, TX, United States, ${ }^{4}$ Community Team, Mapbox, Washington, DC, United States

The spread of pandemics such as COVID-19 is strongly linked to human activities. The objective of this article is to specify and examine early indicators of disease spread risk in cities during the initial stages of outbreak based on patterns of human activities obtained from digital trace data. In this study, the Venables distance $\left(D_{v}\right)$ and the activity density $\left(D_{a}\right)$ are used to quantify and evaluate human activities for 193 United States counties, whose cumulative number of confirmed cases was greater than 100 as of March 31, 2020. Venables distance provides a measure of the agglomeration of the level of human activities based on the average distance of human activities across a city or a county (less distance could lead to a greater contact risk). Activity density provides a measure of level of overall activity level in a county or a city (more activity could lead to a greater risk). Accordingly, Pearson correlation analysis is used to examine the relationship between the two human activity indicators and the basic reproduction number in the following weeks. The results show statistically significant correlations between the indicators of human activities and the basic reproduction number in all counties, as well as a significant leader-follower relationship (time lag) between them. The results also show one to two weeks' lag between the change in activity indicators and the decrease in the basic reproduction number. This result implies that the human activity indicators provide effective early indicators for the spread risk of the pandemic during the early stages of the outbreak. Hence, the results could be used by the authorities to proactively assess the risk of disease spread by monitoring the daily Venables distance and activity density in a proactive manner.

Keywords: COVID-19, early indicators, population activities, time lag relationship, Venables distance, activity density

\section{INTRODUCTION}

The objective of this study is to reveal and evaluate early indicators of human activity during COVID19 period in cities at the initial stages of the outbreak using measures of human activities derived from digital trace data. As an arguably unprecedented global pandemic, the coronavirus disease 2019 (COVID-19) has infected millions of people worldwide with a mortality rate of $6.6 \%$ and a high infection rate (Keni et al., 2020; World Health Organization, 2020). Since the spread of COVID-19 is highly dependent on human activities, incidence of infection could be contained by restricting 
human activities and mobility (Gollwitzer et al., 2020). Many countries and authorities have implemented various nonpharmaceutical interventions (e.g., shelter-in-place orders, regional lockdowns, and travel restrictions), which were undertaken to slow the spread of disease by disrupting transmission chains through restricting human mobility and activities. Such social distancing and activity reduction interventions have proven to be critical in slowing down the spread of pandemics both in previous epidemics (Caley et al., 2008) and during COVID-19 (Anderson et al., 2020; Tian et al., 2020; Li, et al., 2020b; Ramchandani, et al., 2020).

While reduction in human activities is considered an effective measure for containing epidemics and pandemics, there are limited reliable, proven, real-time leading indicators related to human activities that could provide early insights about the risk of disease spread in a region to inform proactive policy making. One reason for this limitation has been the absence of quantitative measures and data that could be examined to proactively evaluate human activities. With advancements in location intelligence data technologies, however, information derived from cellular devices offers a large depository of digital trace data related to human activities increasingly adapted and analyzed to promote understanding of and to quantify human activity and mobility in pandemic analysis, as well as in other applications (Balcan et al., 2009; Asgari et al., 2013; Barbosa et al., 2018). For example, in the context of COVID-19, the radius of gyration, which captures the mobility of individuals using human movement trajectories, was adopted to analyze the COVID-19 spread in Japan (Yabe et al., 2020). Daily step-counts (gathered from smartphones) were used to estimate and predict decreased movement of individuals within the United States during COVID-19 (Gollwitzer et al., 2020). Two of the most important aspects of human activities during an epidemic are agglomeration of activities and intensity of activities.

Although previous research reveals insights regarding human activities in the context of COVID-19, the relationship between human activities and disease-spreading risk has not been fully explored, and leading indicators of human activities to proactively assess the risk of disease spread during the early stages of pandemics are lacking. The majority of research studies (Chang et al., 2020; Cintia et al., 2020; Gao et al., 2020; Li et al., 2020a) focus on quantifying and analyzing the changes in human activities as a consequence of the outbreak of the virus and in response to protective policies (such as shelter-in-place policies). The time-lag relationship between these human activity metrics and the spread of virus, which can be generally described by the basic reproduction number $\left(R_{0}\right)$, has not yet been fully examined. The basic reproduction number, $R_{0}$, is defined as the number of secondary cases produced by one previous case in a completely susceptible population (Dietz, 1993). Although research studies (Lampos et al., 2020; Lu and Reis, 2020) have focused on leading indicators obtained from users' online search behavior, the decrease of online search frequencies may not have direct impact on the spread of the virus. Hence, the previous indicators cannot be utilized for proactive assessment of disease spread risk in a proactive manner. Epidemiological modeling and disease spread modeling are also widely used to predict the spread of the virus (Chen et al., 2020; Ellison, 2020; $\mathrm{Wu}$ et al., 2020). By using fine-grained aggregated human mobility data, Wu et al. (2020) forecasted the subsequent spread of COVID-19 in different geographic regions, with minimal parameterization of the model. Such simulation models could provide the authority handy tools to predict the spread of diseases in the future. The empirical studies and simulation studies are both important for understanding the spread of virus in either the COVID-19 or the future diseases.

In this study, the empirical data related to human activities during COVID-19 was used to reveal the leading relationship between the human activity and the basic reproduction number. We adopted the Venables distance $\left(D_{v}\right)$ index (Louail et al., 2014) and also created the activity density $\left(D_{a}\right)$ index to serve as two recent empirical data indicators to examine the spatial and temporal patterns of human activities across 193 counties in the United States using Mapbox high-resolution temporal-spatial activity index data from January 1 to March 31, 2020. The Venables distance captures the average distance (i.e., concentration) of human activities across a city or county (less distance between persons might indicate a greater contact risk). The activity density captures the intensity level of overall activities in a county or city (higher activity levels might indicate a greater spread risk). Human activities were examined in four categories-social, traffic, work, and other-based on the location and time of activities. Accordingly, we analyzed the correlation between the two metrics $\left(D_{v}\right.$ and $\left.D_{a}\right)$ and the basic reproduction number for 193 counties with the highest number of confirmed COVID-19 cases. The significant correlation between the two population activity measures and the extent of spread of the virus suggested that these two measures at the beginning stage of the outbreak could provide promising leading indicators of the risk of spread based on human activity. The examination of population activity measures as leading indicators of pandemic spread risk is critical for situational awareness and monitoring and would complement the insights obtained from standard mathematical disease spread models. Although the spread of the pandemic is a complex phenomenon and is affected by various factors, the focus of this research is only on the human activity aspect and early indicators related to human activity.

The rest of this article is organized into three sections. The first section discusses the description of the two datasets (Mapbox data and total confirmed cases number data), as well as the analysis methods. The second section describes the results of time-lag correlation analysis between the two metrics and the basic reproduction number. The last section presents the results and the implications of the findings for future work.

\section{METHODS}

In this section, we describe the two datasets-Mapbox data and total confirmed cases number data-and the procedures for human activity categorization. Also covered in this section are definitions and equations related to the Venables distance $\left(D_{v}\right)$, the activity density $\left(D_{a}\right)$, and the basic reproduction number $\left(R_{0}\right)$. The time-lag 


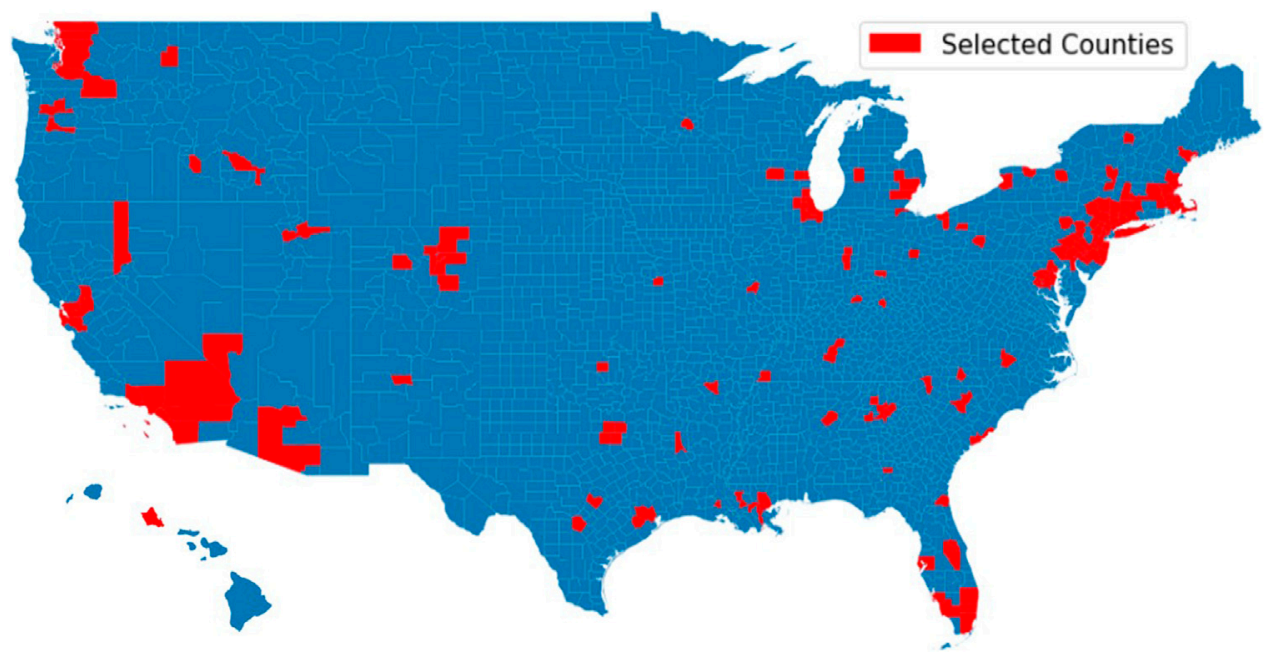

FIGURE 1| 193 selected counties whose cumulative confirmed cases were greater than 100 as of March 31, 2020

cross-correlation analysis method is presented at the end of this section.

\section{Data Source and Preprocessing}

We utilized digital trace telemetry data obtained from Mapbox from January 1 to March 30, 2020. The dataset contains a metric of telemetry-based human activity, $a_{T, t}$, which varies across spatial tiles and time $t$. The partition of tiles is based on Mercantile, a Python library, which is capable of creating spatial-resolution grids all worldwide. The $a_{T, t}$ is collected, aggregated, and normalized by Mapbox from geography information updates of users' cell phone locations by time flows. The more users located in a tile at time $t$, the higher the human activity (i.e., $a_{T, t}$ ). The dataset comprises the United States and the District of Columbia; however, in this study, we examined only 193 counties whose cumulative confirmed cases were greater than 100 as of March 31, 2020 (Figure 1). In the raw data, the temporal resolution is $4 \mathrm{~h}$. Each tile represents about 100 by 100 square meters for spatial resolution. Since the data is derived from cell phone activity, data may not exist for all cells at all times. For example, a park open during the day but closed at night would not generate any data at midnight. Also, for protecting users' privacy and the data aggregation process, tiles with a small number of users are reported without any activity data. It is also noteworthy that the data is aggregated and normalized for each month, so the absolute values of activity indices for different months cannot be directly compared.

To reveal the time-lag relationship between metrics and spread of the virus, the total number of confirmed cases was used. We obtained the data from the COVID-19 Data Repository by the Center for Systems Science and Engineering (CSSE) at Johns Hopkins University (2020). The data in this repository were gathered and aggregated from various sources, such as the World Health Organization (WHO) and the Centers for Disease Control and Prevention (CDC). We extracted the total number of confirmed cases $c_{i, j}$ from the CSSE repository, where $i$ represents each date and $j$ represents each county.

\section{Tile Categorization}

The nature of an activity might put its participants at a higher risk level for contracting the virus. For example, activities in public common areas, such as grocery stores or gyms, would lead to greater risk of disease spread compared to the activities in residential areas, such as working from home or walking a dog in the community. The fine granularity of the spatial resolution enables classification of each tile into one of the four categories: (1) social tiles, (2) traffic tiles, (3) work tiles, and (4) other tiles. Categorization is based on the following characteristics: (1) social tiles are the location of at least one point of interest location; the location information of point of interest is extracted from SafeGraph data (including restaurant, gas station, and commercial complex) (SafeGraph, 2020); (2) traffic tiles are extracted by mapping the traffic network with all tiles including roads; (3) work tiles are identified based on lack of activity during the late evening hours; and (4) other tiles are located in residential areas. We assigned these four tags to each tile from social, traffic, work to other. Once a tile is assigned with a tag, it is excluded for further categorization. We categorized tiles in this way to examine the importance of human activity in each category and its relationship with the reproduction number. The analysis in this research examines human activities for social, work, and traffic tiles separately. All the residential tiles are excluded from the analysis because the human activities in these tiles have less influence on the contact level among people. Example tile maps related to each category are shown in Figure 2 for Harris County, Texas.

\section{Venables Distance $\left(D_{v}\right)$}

To quantify the agglomeration of human activities, we used the Venables distance $\left(D_{v}\right)$ as a weighted average distance of human activities. The Venables distance aggregates the spatial 


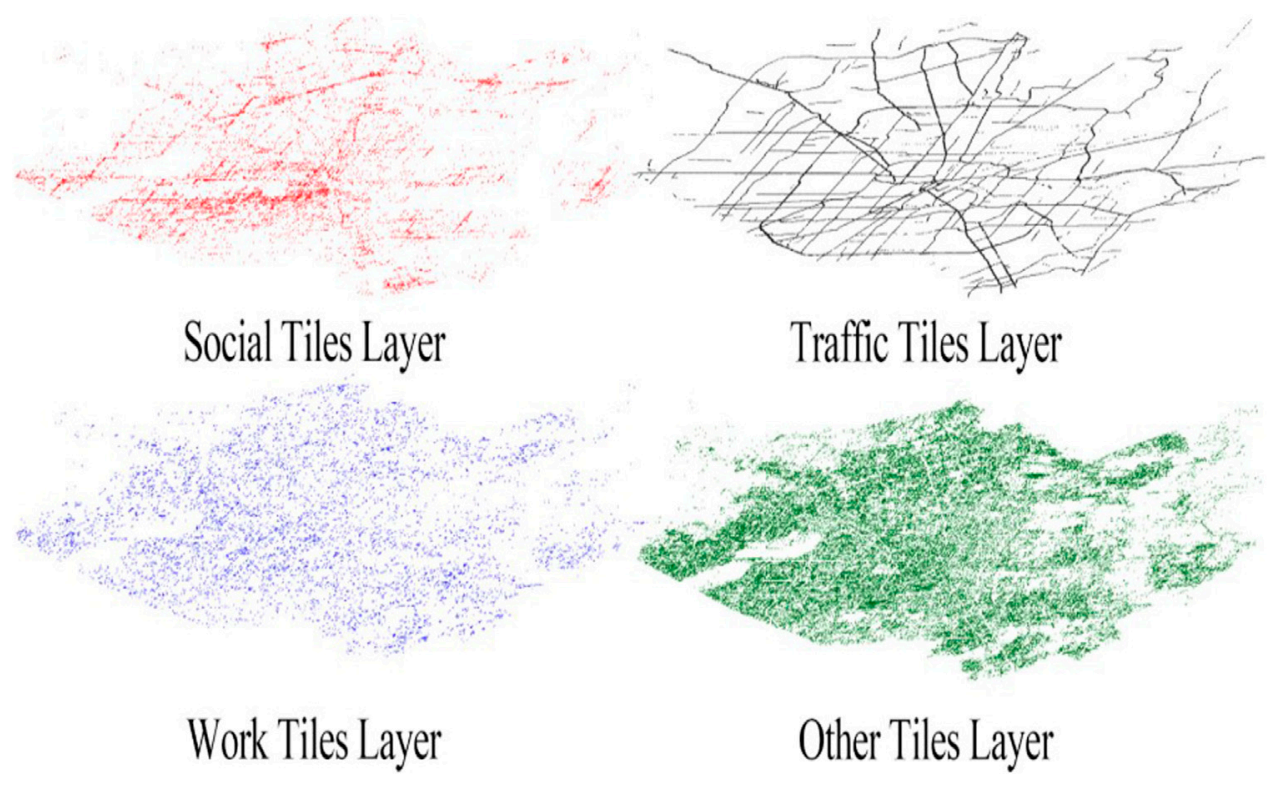

FIGURE 2 | Maps of four different tile categories (social, traffic, work, and other) in Harris County, Texas.

distribution of $a_{T, t}$ in a county and captures the urban spatial structure of human activities (Louail et al., 2014). The Venables distance is a metric to describe how distant the human activities are from each other. It also provides a metric for concentration activities. In examining the risk of pandemic, understanding the concentration of human activities is an important consideration. Hence, the Venables distance provides a metric to examine fluctuations in this aspect of human activities. Compared with the well-known spatial autocorrelation metric, the meaning of $D_{v}$ is easier to be explained and understood. Also, the meaning of $D_{v}$ is different from that of spatial autocorrelation, which describes the presence of systematic in spatial space. The value of spatial autocorrelation is from -1 to 1 , and it could be negative if the adjacent observations tend to have very contrasting values. The Venables distance, however, is a metric to describe how far the human activities are apart from each other, and its unit, kilometer, has reality meaning. The $D_{v}$ is calculated using Eq. 1:

$$
D_{v}(t)=\frac{\sum_{T_{1} \neq T_{2}} a_{T_{1}, t} \cdot a_{T_{2}, t} \cdot d_{T_{1}, T_{2}}}{\sum_{T_{1} \neq T_{2}} a_{T_{1}, t} \cdot a_{T_{2}, t}}
$$

where $a_{T_{1}, t}$ and $a_{T_{2}, t}$ are the metrics of human activities in Tile and Tile $e_{2}$ at time $t$, respectively, and $d_{T_{1}, T_{2}}$ is the distance from the centroids between these two tiles. In Harris County, Texas, there are more than $70 \mathrm{~K}$ unique tiles, which makes it computationally expensive to analyze all pairs of existing tiles. To reduce the computational burden, we aggregated the 100-by-100 squaremeter tiles, $a_{T, t}$, to square cells $2 \mathrm{~km}$ in length using Eq. 2:

$$
a_{k, t}=\frac{\sum_{\text {for all } T \text { in cell } k} a_{T, t}}{A_{k}},
$$

where $a_{k, t}$ is the intensity of human activity in cell $k$, at time $t$ and $A_{k}$ is the area of the cell $k$. By aggregating human activity into a larger spatial cell, we reduced the computational efforts, maintaining a meaningful spatial resolution without losing important characteristics in the raw data. Accordingly, the modified Venables distance is derived as shown in Eq. 3:

$$
D_{v}(t)=\frac{\sum_{k_{1} \neq k_{2}} a_{k_{1}, t} \cdot a_{k_{2}, t} \cdot d_{k_{1}, k_{2}}}{\sum_{k_{1} \neq k_{2}} a_{k_{1}, t} \cdot a_{k_{2}, t}},
$$

where $a_{k_{1}, t}$ and $a_{k_{2}, t}$ are the intensity of human activities in cells $k_{1}$ and $k_{2}$ at time $t$, respectively, and $d_{k_{1}, k_{2}}$ is the distance from the centroids between these two cells. In Eq. 3, the values of the activity intensity $\left(a_{k, t}\right)$ are used as weights to calculate a human-activity-weighted distance for the whole area. In other words, the relative values of $a_{k, t}$ were used to examine changes in agglomeration of activities. We calculated $D_{v}(t)$ for each county $j$, which is denoted as $D_{v}(j, t)$ for all cells in the county $j$. Due to high computational cost of conducting, we aggregated the data into $2 \mathrm{~km}$ square. The Venables distance describes how human activities are apart from each other, and the spatial resolution of $2 \mathrm{~km}$ is capable to capture such feature.

\section{Activity Density $\left(D_{a}\right)$}

Although $D_{v}$ captures the agglomeration of human activities, the density of activities is also critical for examining population contact. To make the raw data $\left(a_{T, t}\right)$ from Mapbox comparable among different months, we denormalized the activity index to the contact activity metric $c a_{T, t}$ for each tile and each month (where $t$ is time). The original human activity data was obtained from the use of cell phones and was normalized within each month across all tiles and time windows. Every value above the 99.9 percentile is set to 1 , and every value below that is scaled linearly. Since the maximum 

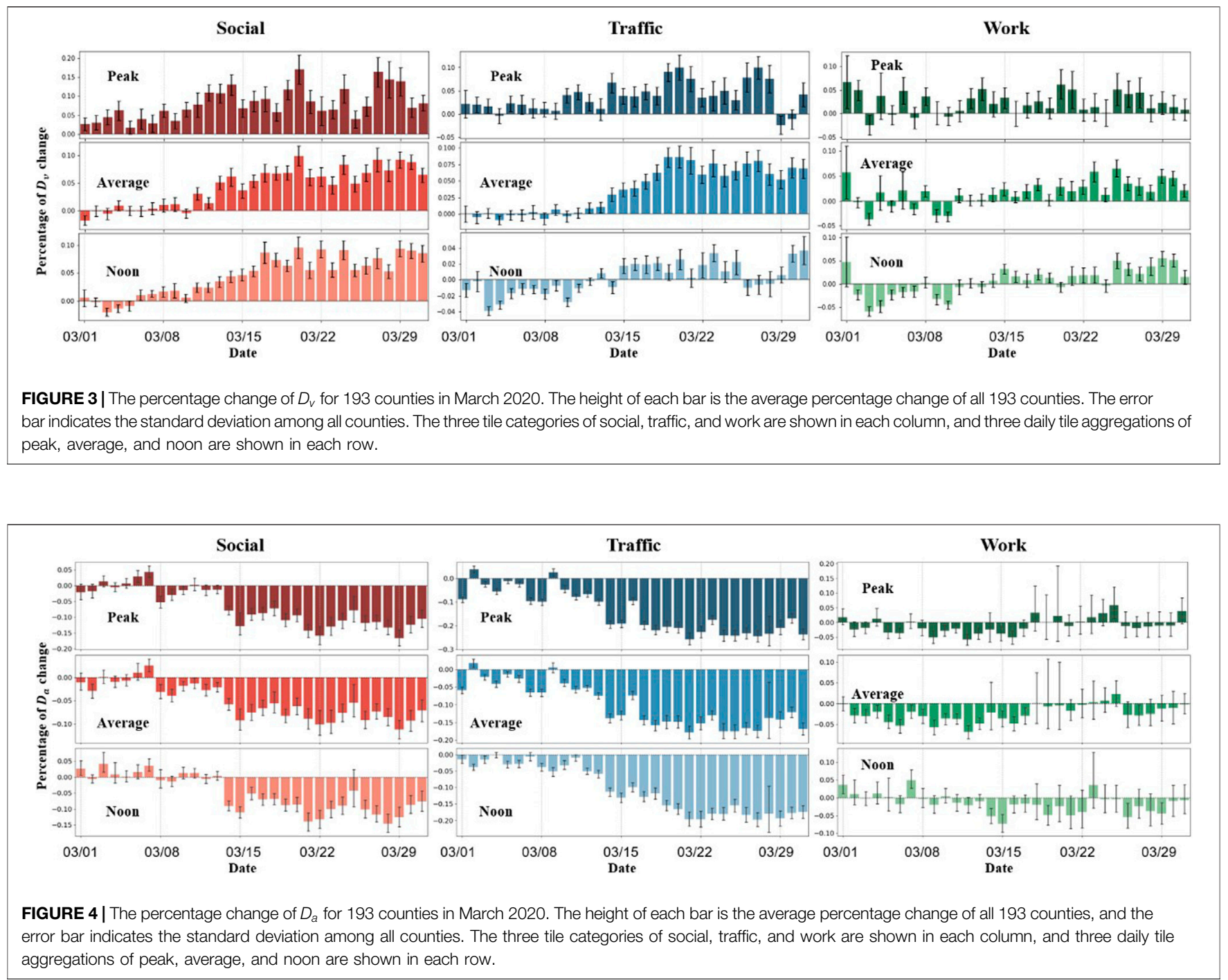

value of human activities across the entire United States (the denominator of the normalization process) could be different in different months, the normalized data cannot be compared across different months. In the denormalization process, the assumption was that, in each month, the minimum human activity intensity among tiles is the same. First, the tile with minimum human activity index $\left(a_{T, t}\right)$ in each month was found. Then we set the value of contact activity $c a_{T, t}$ in that low-activity tile as 5 and denormalize the values for other tiles based on this value. The value of tiles with the lowest activity is set in order to keep the minimum activity in the tiles across the United States fixed and consistent. By doing so, we can denormalize the data uniformly for all other tiles in the country. For each county, the activity density at time $t\left[D_{a}(t)\right]$ was calculated using Eq. 4:

$$
D_{a}(t)=\sqrt{\frac{1}{N} \sum_{T=1}^{N} c a_{T, t}^{2}} .
$$

\section{Basic Reproduction Number Estimation}

The basic reproduction number $\left(R_{0}\right)$ (the number of secondary cases arising from one previous case) is a critical parameter in epidemic modeling for understanding the speed of disease spread (Dietz, 1993; Gatto et al., 2020), as well as the risk of virus spread (Newman, 2002; Liu et al., 2018; Aleta et al., 2020; Giordano et al., 2020). We adopted the R0 in the analysis, assuming a complete susceptibility of the population. This is because, at the early stage of the COVID-19 pandemic (March, 2020), all people were susceptible. The effective reproduction number $\left(R_{t}\right)$ is another metric which is widely used to describe the spread speed of disease (Nishiura and Chowell,, 2009; Althaus 2014). $R_{t}$ assumes only partial population is susceptible and takes into account the effect of policies such as social distancing in measuring the disease spread. Thus, $R_{t}$ can be used in cases with populations having immune members (Delamater et al., 2019). Hence, $R_{t}$ would be more appropriate in analyses at the later stage of the pandemic. In the early stage of COVID-19, it was a novel virus for all people and everyone was susceptible. Hence the basic reproduction 

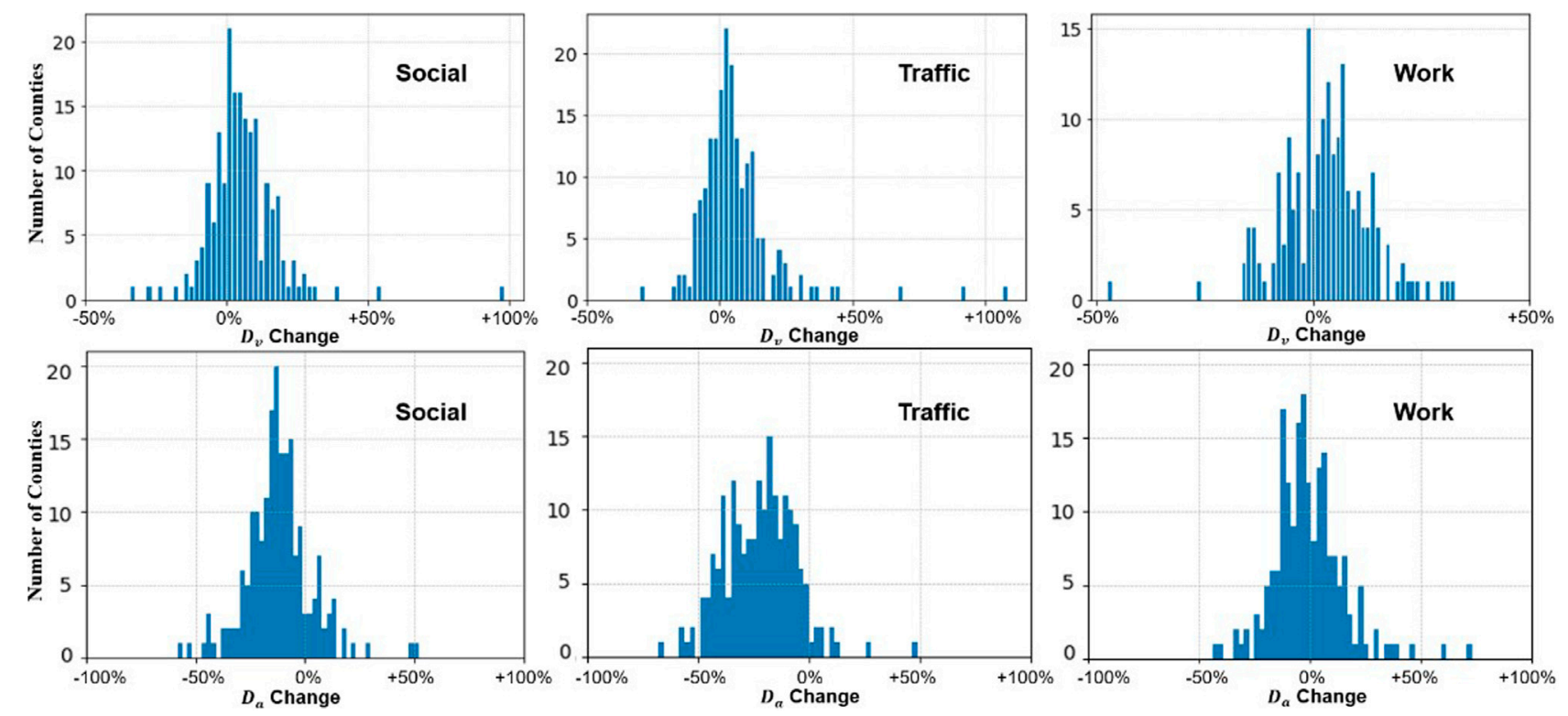

FIGURE 5 | Histogram plots of average percentage change of $D_{v}$ and $D_{a}$ (each row) for three different categories (each column).

number $\left(R_{0}\right)$ was used in this study to represent the spread speed of the virus. $R_{0}$ is calculated using Eq. 5:

$$
c_{i+t}=c_{i} \cdot R_{0}^{t / \tau},
$$

where $c_{i}$ and $c_{i+t}$ represent the total confirmed cases in day $i$ and day $i+t$, respectively, and $\tau$ is a constant parameter. We estimated $R_{0}$ using CDC data $\left(c_{i, j}\right)$ in Eq. 6 :

$$
R_{0, j}=e^{\frac{\ln c_{i, j}-\ln c_{i-t, j}}{t}},
$$

where $R_{0 i, j}$ is the basic reproduction number at date $i$ in county $j$. Because the CDC total confirmed cases data fluctuates within the course of a week (i.e., more reported cases in weekdays and less reported cases during weekends), the time interval $t$ was set to 7 days. Based on the existing literature and simulation models related to COVID-19 (Zhang et al., 2020a; Zhang et al., 2020b; Fan et al., 2020), the constant parameter $\tau$ was set to 5.1 days. Accordingly, the $R_{0}$ was calculated for all 193 counties for the analysis period. The daily basic reproduction number for each county will be used to conduct the correlation analysis with proposed two indicators of human activities.

\section{Time Lagged Cross-Correlation Analysis}

In the next step, we examined the correlation between the two human activity indicators and the basic reproduction number across all counties. Since these variables are a time series, we used time-lagged cross-correlation analysis to assess the synchrony of time series data sets. The cross-correlation coefficient was calculated using Eq. 7:

$$
\rho_{A_{1} A_{2}}(\tau)=\frac{\operatorname{Cov}\left(A_{1}(t), A_{2}(t+\delta)\right)}{\sigma_{A_{1}} \sigma_{A_{2}}},
$$

where $\rho_{A_{1} A_{2}}$ is the cross-correlation coefficient for two time series data $A_{1}$ and $A_{2} ; \delta$ is the time offset of $A_{2} ; \operatorname{Cov}(X, Y)$ is the function calculating the covariance of two variables; $\sigma_{A_{1}}$ and $\sigma_{A_{2}}$ are the standard deviation of data $A_{1}$ and $A_{2}$, respectively. Based on the definition, $\rho_{A_{1} A_{2}}$ represents the correlation between two variables and $\left|\rho_{A_{1} A_{2}}\right| \leq 1 \quad\left(\left|\rho_{A_{1} A_{2}}\right|=1\right.$ happens if and only if $A_{1}=m A_{2}+n$, where $\mathrm{m}$ and $\mathrm{n}$ are constants). Then, by iteratively calculating the $\rho_{A_{1} A_{2}}(\delta)$ with different $\delta$, the correlation coefficient would reach its peak when $\delta=T$, and $T$ was determined as the time lag between two variables.

\section{RESULTS}

This section presents the results related to the calculation of the two human activity metrics and their time-lagged correlation with the basic reproduction number across 193 counties during the initial stage of the COVID-19 outbreak in the United States.

\section{Evaluation of Human Activity for Each Category Among Counties}

In this study, the Venables distance $\left(D_{v}\right)$ and the activity density $\left(D_{a}\right)$ were calculated to assess the human activities at the county level using data from Mapbox. The very first four weeks (January $1-28$ ) were considered as the baseline, and $D_{v}$ and $D_{a}$ values were compared with the average baseline in corresponding weekdays. For example, the $D_{v}$ values on March 1 (Sunday) were compared with the mean value of $D_{v}$ values on Sundays between January 1 and 28. Three different ways of daily activity aggregation were used: peak, average, and noon. The peak (largest), average, or the noon (11 a.m.-3 p.m.) values of human activities $a_{\text {tile, } t}$ were selected and set as the representative value of each tile at each day. Figure 3 and Figure 4 show the percentage of $D_{v}$ and $D_{a}$ 

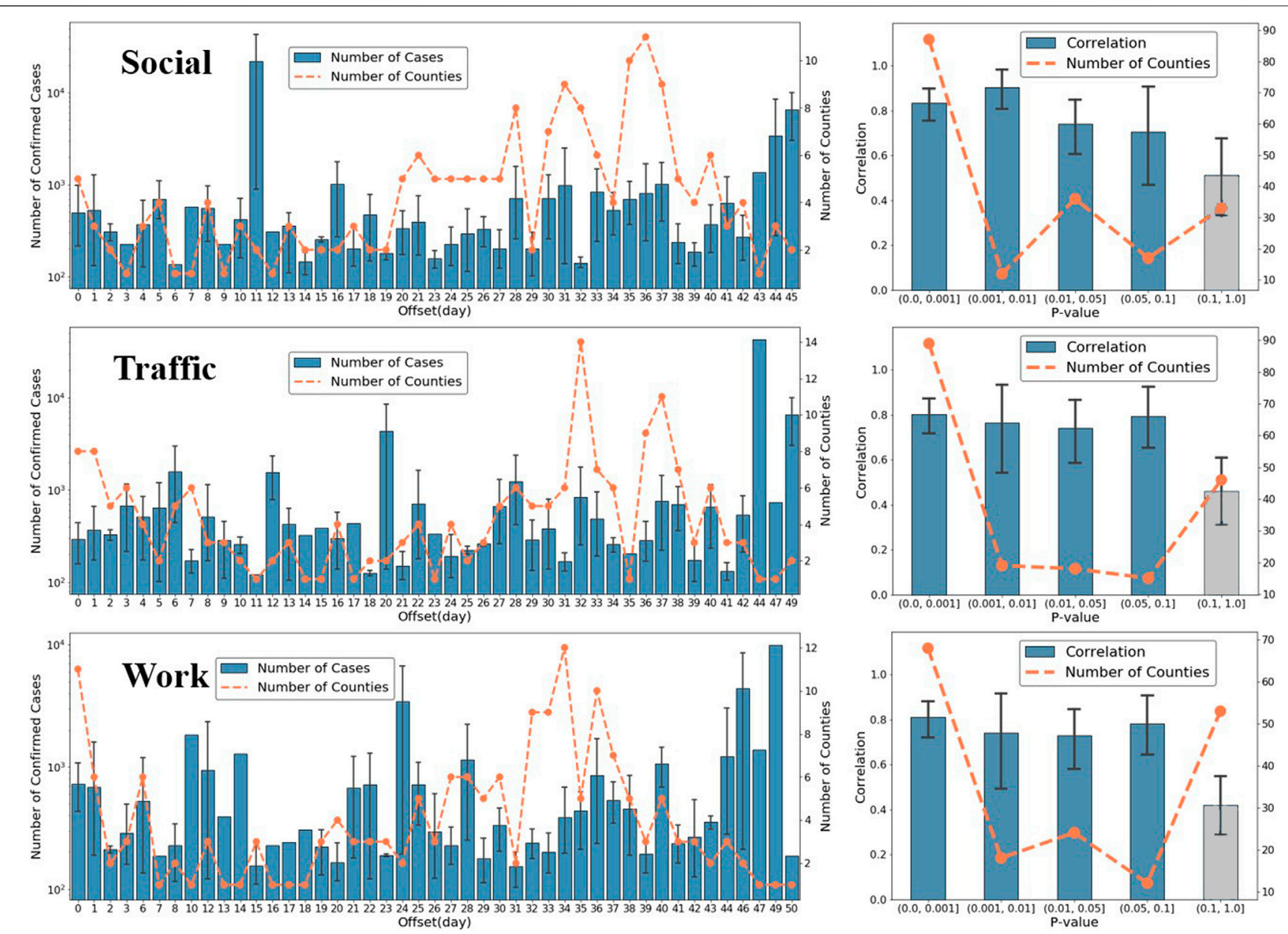

FIGURE 6 | The time-lagged correlation analysis between Venables distance $\left(D_{v}\right)$ change and reproduction number $\left(R_{0}\right)$. The left column shows the number of cases and the number of counties for different offset days, and the right column shows the correlation index and the number of counties with different $p$-value intervals. Each row presents one of the three tile activity categories: social, traffic, and work.

change for social, traffic, and work activity categories and for three types of daily tile aggregation.

The increasing trend of Venables distance $\left(D_{v}\right)$ implies declining concentration and rising distance among people, and the decreasing trend of activity density $\left(D_{a}\right)$ indicates less human activity compared with the beginning of this year. Due to the shelterin-place policies, residents changed their daily activity patterns. More and more people reduced the nonessential outdoor activities (e.g., shopping in supermarkets, exercising in gyms, and eating at restaurants). Such changes in daily human activity patterns led to the change of $D_{v}$ and $D_{a}$. For the three categories, significant change can be seen in both social and traffic tiles, while the change in work tiles is not obvious. This is because the activities in work tiles could be essential activities. The $D_{v}$ increased the most, around $15 \%$, in social tiles, while the $D_{a}$ fell the most in traffic tiles, which is around $25 \%$. Differences among the three types of daily tile aggregation were not significant. The percentage change of peak values is slightly greater than the other two values, indicating that peak values are influenced more by COVID-19, while average values are more stable. The following analysis uses peak values to calculate $D_{v}$ and $D_{a}$.

Histograms of average percentage change of $D_{v}$ and $D_{a}$ for each county are shown in Figure 5. The average percentage change is calculated during the last week of March. The $D_{v}$ values in the majority of counties increased, and $D_{a}$ values decreased for social and traffic categories, while the work category shows more even distribution around $0 \%$ for both $D_{v}$ and $D_{a}$. These histogram plots are consistent with the claim that human activities in work tiles are more essential than the other two (social and traffic), which did not show significant change during the COVID-19 study period.

While $D_{v}$ and $D_{a}$ describe the different global characteristics of human activity-the $D_{v}$ captures spatial distribution of human activity and the $D_{a}$ focuses on the intensity of human activity-they both reveal the insight of massive human activity patterns, which could have a quite significant influence on the spread of the virus. The correlation 


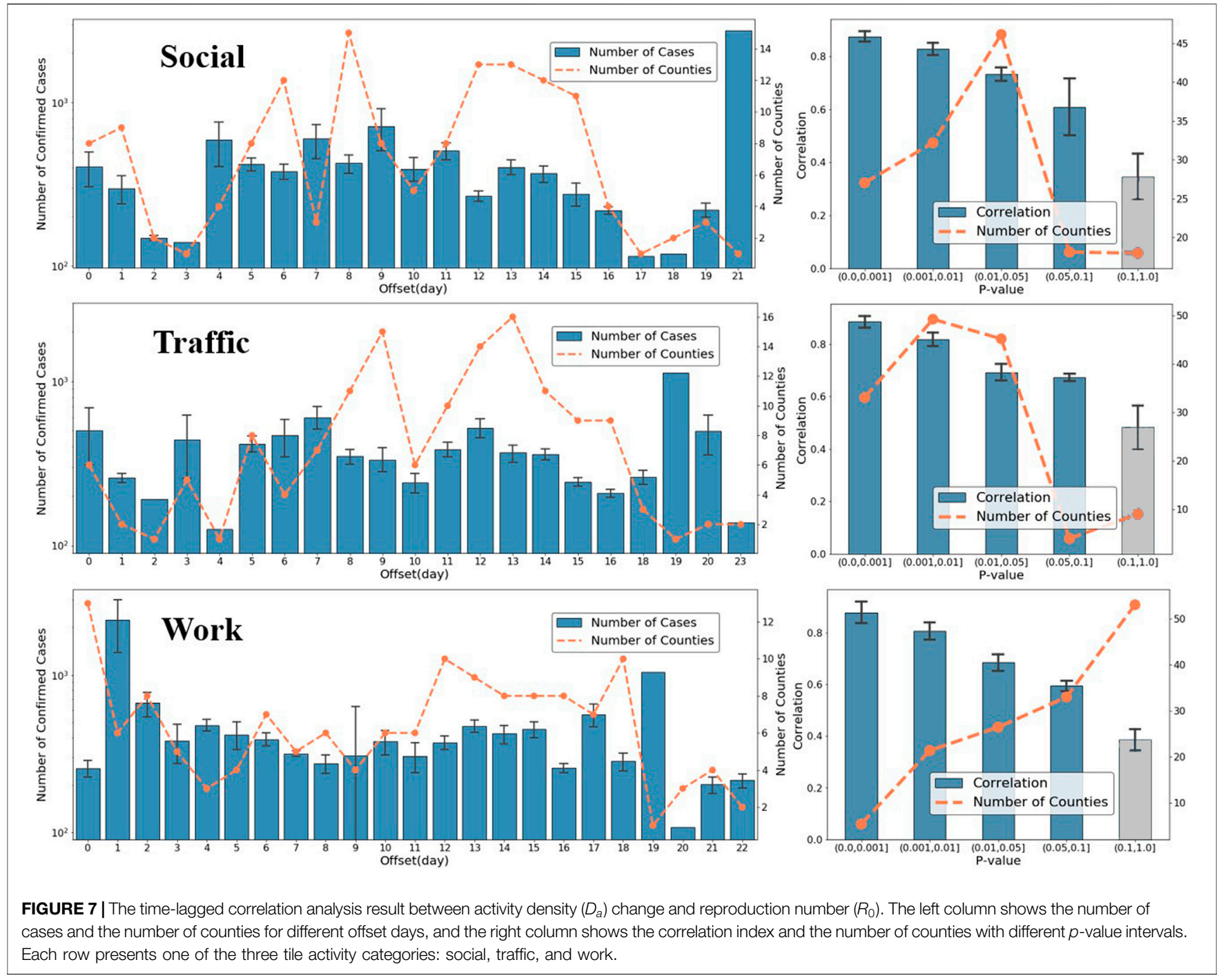

analysis between these two metrics and the basic reproduction number $R_{0}$ becomes critical.

\section{TIME-LAGGED CORRELATION ANALYSIS}

The spread of the coronavirus is closely related to the human activity patterns. In the previous section, we showed that the average distance between human activities $\left(D_{v}\right)$ increased by $10 \%-15 \%$, and the average human activity intensity $\left(D_{a}\right)$ decreased by $5 \%-10 \%$ for social tiles during March 2020 compared with the baseline period of January 2020. This result provides a good indication of the reduction in human activities in response to social distancing policies. In this section, we examine the extent to which the change in human activity metrics was related to the change in reproduction rate of coronavirus in the 193 counties under study. Hence, we conducted the time-lagged correlation analysis for the two human activity metrics calculated based on social, traffic, and work tile activity categories. Then all of the counties were grouped by the time lags. For each group, we counted the number of counties and summed the confirmed cases numbers. We plotted both the number of confirmed cases and the number of counties in Figures 6, 7 to show what the time lag is for most counties and for the most confirmed cases. The range of the confirmed cases numbers for different counties is large. The minimum confirmed cases number is 101, and the maximum number is almost 10,000 in New York Counties. That is why we used both of them to represent the result in a more comprehensive way. Figure 6 shows the time offset results between the Venables distance $\left(D_{v}\right)$ change and the basic reproduction number $\left(R_{0}\right)$ for social, traffic, and work activity categories. Since the number of confirmed cases follows a skewed distribution during March 2020, the log scale is used to illustrate the results. The results show that, in majority of typical counties, the decline in the basic reproduction number $\left(R_{0}\right)$ happens 20-40 days after the increase in Venables distance. This result is consistent across all the three activity categories. In the right column of Figure 6, the bar charts show the correlation between the offset $D_{v}$ and $R_{0}$ within different $p$-value intervals. The average correlation coefficients (with $p$-values less than 0.05 ) 

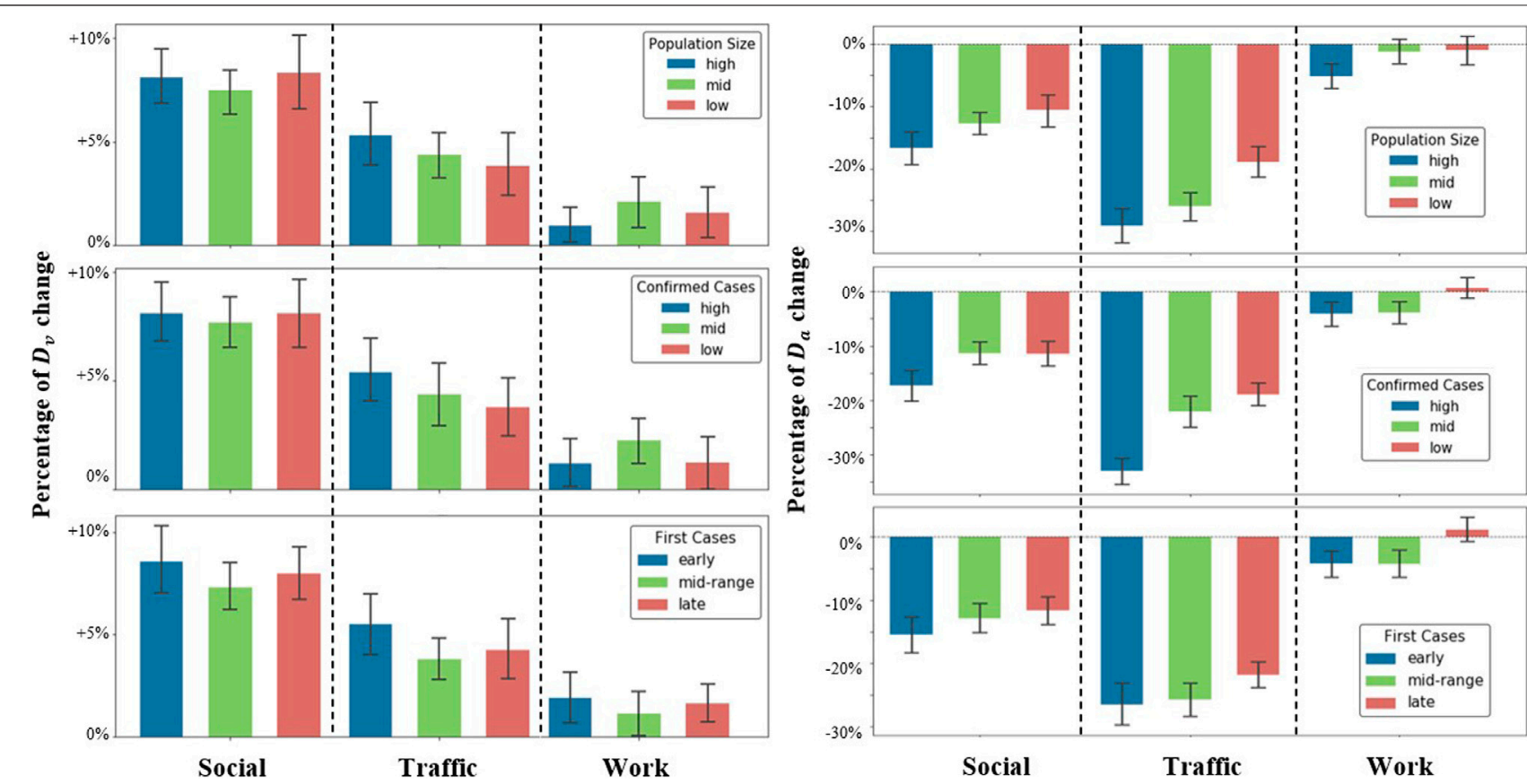

FIGURE 8 | Change in Venables distances $\left(D_{v}\right)$ and activity density $\left(D_{a}\right)$ across counties with different population sizes, confirmed cases number, and the date of first confirmed case in the three tile activity categories (social, traffic, and work).

are around 0.8 for each category, indicating a significant relationship between the increased distance among human activities and the decline in the virus spread speed. For the $p$-values greater than 0.05 (indicating no sufficient evidence to prove the correlation between two variables), the correlation indices are correspondingly smaller. The number of counties in each $p$-value interval shows that about $50 \%$ of the counties have $p$-values less than 0.001 for Venables distance calculated based on social and traffic activity tiles. The results related to work tiles, however, show a significant correlation between the two variables in a smaller number of counties.

Figure 7 shows the time offset result between activity density $\left(D_{a}\right)$ change and reproduction number $\left(R_{0}\right)$ for the three activity categories. The results show that the decline in the basic reproduction number happens 6-17 days after the reduction of the activity intensity $\left(D_{a}\right)$; a similar result exists in all activity categories. The time lag is less than the one obtained for the Venables distance $\left(D_{v}\right)$, which means that the spread of the virus responds to human activity intensity reduction more quickly than to human agglomeration reduction. In the right column of Figure 7, the bar charts show the correlation of offset $D_{a}$ and $R_{0}$ in different $p$-value intervals. The average correlation indices for $p$-values less than 0.05 are around 0.9 for tile activity categories. This result indicates a significant relationship between the human activity intensity reduction and the decline in the virus spread speed. For $p$-values greater than 0.05 , the correlation indices are smaller as well. The number of counties in each $p$-value interval shows that about $50 \%$ of counties have $p$-values less than 0.01 for social and traffic tiles, while the work tiles result shows $p$-values between 0.1 and 1.0 (indicating a nonsignificant correlation).

\section{Heterogeneity for Different County Features}

In the next step, we examined the variation of findings across counties with different population sizes, number of confirmed cases, and date of first confirmed cases. The goal is to examine the extent to which the correlation between the two metrics of human activities and the reproduction number is sensitive to these county features. The 193 counties were divided into three uniform categories according to population size and confirmed cases (on March 18, 2020) labeled high, medium, and low. Similarly, the first case dates were labeled as early, mid-range, and late for each one-third of counties. Then, the changes in $D_{v}$ and $D_{a}$ (on March 31,2020) were examined for each label in each tile category, and the results are plotted in Figure 8. $D_{v}$ and $D_{a}$ show different heterogeneities facing these county features. In the left side of Figure 8, we cannot detect a clear difference in $D_{v}$ value changes in different group. In the right side of Figure 8, however, we can observe that $D_{a}$ values decreased more in the counties with larger population size, higher confirmed cases number, and earlier first confirmed case, and such pattern exists for all the social, traffic, and work tiles. Based on the definition, $D_{a}$ represents the intensity of human activities. Based on this result shown in Figure 8, we can conclude that the intensity of human activities decreased more in counties with larger population size, higher confirmed cases number, and earlier first confirmed cases, which could imply a greater recognition and response to the pandemic risks in such counties. 


\section{DISCUSSION}

This study shows the utility of two human activity metrics [the Venables distance $\left(D_{v}\right)$ and the activity density $\left.\left(D_{a}\right)\right]$ as leading indicators of human activity for the spread speed of COVID-19 in the early stages of the outbreak. These metrics were derived from digital trace data obtained from Mapbox high-resolution temporal-spatial datasets. The results provide statistical evidence regarding the time-lag correlation between these two metrics and the basic reproduction number $\left(R_{0}\right)$ in the context of COVID-19. The results regarding the significant leader-follower relationship between human activities and the rate of spread of viral infections could be used by the public health officials and decision makers to monitor human activity and provide insights regarding the trends in the basic reproduction number in the future one to two weeks. For example, time lag indicates that the spread of the virus responds to human activity intensity reduction more quickly than to human agglomeration reduction. As for the heterogeneity of indicators, the result shows that the intensity of human activities decreased more in the counties with more population, more confirmed cases, and earlier first confirmed cases, which indicates a greater recognition and response to the pandemic risks in these counties.

There exist other studies that examined human mobility in the context of COVID-19. Wang et al. (2020) examined the similar time-lag effect of human mobility on the COVID-19 infections in the 80 cities most affected in China from Jan 17 to Feb 29. The results showed that the time lag is about 10 days. The index of intracity traffic volume (provided by Baidu) was used to represent the human mobility. Such highly aggregated data, however, may lose some critical spatial information about human activities. Xiong et al. (2020) analyzed mobile device data at each United States county for the COVID-19 period. The origindestination travel demand and aggregate mobility inflow were used to represent the human activities, and the results showed the dynamics in a positive relationship between human mobility and COVID-19 transmission. Compared with the mentioned similar metrics describing human activities, the two indicators in this article $\left(D_{v}\right.$ and $\left.D_{a}\right)$ capture both the agglomeration of activities and intensity of activities, which are two of the most important aspects of human activities during an epidemic. The $D_{v}$ and $D_{a}$ are also easy to calculate based on the human activity data provided by Mapbox. They are the early indicators for authority to monitor in advance the spread of the virus in the future.

This study has some limitations which need to be improved in future studies. First, the tile activity categorization-social, work, and traffic-is not precise. One tile could be labeled as both social

\section{REFERENCES}

Aleta, A., Martin-Corral, D., y Piontti, A. P., Ajelli, M., Litvinova, M., Chinazzi, M., et al. (2020). Modeling the impact of social distancing, testing, contact tracing and household quarantine on second-wave scenarios of the COVID-19 epidemic. medRxiv [Preprint]. Available at: https://doi.org/10.1101/2020.05. 06.20092841 (Accessed May 18, 2020).

Althaus, C. L. (2014). Estimating the reproduction number of Ebola virus (EBOV) during the 2014 outbreak in West Africa. PLoS Curr 6. doi:10.1371/currents. outbreaks.91afb5e0f279e7f29e7056095255b288 and work. In this study and due to data availability limitations, however, we classified tiles into only one of the three categories. Second, the CDC confirmed-cases data had limitations due to testing availability. In this study, we did not adjust the confirmed case data based on the extent of testing in different counties. A lack of testing in some areas resulted in the underestimation of the total cases.

\section{DATA AVAILABILITY STATEMENT}

The data analyzed in this study is subject to the following licenses/restrictions: the data that support the findings of this study are available from Mapbox, but restrictions apply to the availability of these data, which were used under license for the current study and so are not publicly available. Requests to access these datasets should be directed to Mikel Maron, mikel@mapbox.com.

\section{AUTHOR CONTRIBUTIONS}

Research design and conceptualization were carried out by XG, $\mathrm{CF}$, and AM; data collection, processing, analysis, and visualization were performed by XG, CF, YY, SL, and QL; writing was done by $\mathrm{XG}$ and $\mathrm{AM}$; reviewing and revising were made by all authors.

\section{FUNDING}

This work was supported by several grants including the United States National Science Foundation RAPID project \#2026814, Urban Resilience to Health Emergencies: "Revealing Latent Epidemic Spread Risks from Population Activity Fluctuations and Collective Sense-making," and Microsoft AI for Health COVID-19 Grant for cloud computing resources.

\section{ACKNOWLEDGMENTS}

The authors would also like to acknowledge that Mapbox provided digital trace telemetry data of human activity and that SafeGraph provided POI data. The authors would like to thank Kieran Gupta, Sofia Heisler, and Ruggero Tacchi from Mapbox for providing technical support.

Anderson, R. M., Heesterbeek, H., Klinkenberg, D., and Hollingsworth, T. D (2020). How will country-based mitigation measures influence the course of the COVID-19 epidemic? Lancet 395 (10228), 931-934. doi:10.1016/S01406736(20)30567-5

Asgari, F., Gauthier, V., and Becker, M. (2013). A survey on human mobility and its applications. arXiv [Preprint]. Available at: https://arxiv.org/abs/1307.0814 (Accessed Jul 2, 2013).

Balcan, D., Colizza, V., Gonçalves, B., Hu, H., Ramasco, J. J., and Vespignani, A. (2009). Multiscale mobility networks and the spatial spreading of infectious diseases. Proc. Natl. Acad. Sci. United States 106 (51), 21484-21489. doi:10. 1073/pnas.0906910106 
Barbosa, H., Barthelemy, M., Ghoshal, G., James, C. R., Lenormand, M., Louail, T., et al. (2018). Human mobility: models and applications. Phys. Rep. 734, 1-74. doi:10.1016/j.physrep.2018.01.001

Caley, P., Philp, D. J., and McCracken, K. (2008). Quantifying social distancing arising from pandemic influenza. J. R. Soc. Interface 5 (23), 631-639. doi:10. 1098/rsif.2007.1197

Chang, S. Y., Pierson, E., Koh, P. W., Gerardin, J., Redbird, B., Grusky, D., et al. (2020). Mobility network modeling explains higher SARS-CoV-2 infection rates among disadvantaged groups and informs reopening strategies. medRxiv [Preprint]. Available at: https://doi.org/10.1101/2020.06.15.20131979 (Accessed Aug 14, 2020).

Chen, Y. C., Lu, P. E., Chang, C. S., and Liu, T. H. (2020). A Time-dependent SIR model for COVID-19 with undetectable infected persons. IEEE Transactions on Network Science and Engineering 7 4. arXiv [Preprint]. Available at: https:// arxiv.org/abs/2003.00122 (Accessed Apr 28, 2020).

Cintia, P., Fadda, D., Giannotti, F., Pappalardo, L., Rossetti, G., Pedreschi, D., et al. (2020). The relationship between human mobility and viral transmissibility during the COVID-19 epidemics in Italy. arXiv [Preprint]. Available at: https:// arxiv.org/abs/2006.03141 (Accessed June 4, 2020).

Delamater, P. L., Street, E. J., Leslie, T. F., Yang, Y. T., and Jacobsen, K. H. (2019). Complexity of the basic reproduction number (R0). Emerg. Infect. Dis. 25 (1), 1. doi:10.3201/eid2501.171901

Dietz, K. (1993). The estimation of the basic reproduction number for infectious diseases. Stat. Methods Med. Res. 2 (1), 23-41. doi:10.1177/096228029300200103

Ellison, G. (2020). Implications of heterogeneous SIR models for analyses of COVID19. Massachusetts, MA: National Bureau of Economic Research, Working paper No. w27373. doi:10.3386/w27373

Fan, C., Lee, S., Yang, Y., Oztekin, B., Li, Q., and Mostafavi, A. (2020). Effects of population co-location reduction on cross-county transmission risk of COVID19 in the United States. arXiv [Preprint]. Available at: https://arxiv.org/abs/ 2006.01054. (Accessed Jun 1, 2020).

Gao, S., Rao, J., Kang, Y., Liang, Y., and Kruse, J. (2020). Mapping county-level mobility pattern changes in the United States in response to COVID-19. Sigspatial Special 12 (1), 16-26. doi:10.1145/3404111.3404115

Gatto, M., Bertuzzo, E., Mari, L., Miccoli, S., Carraro, L., Casagrandi, R., et al. (2020). Spread and dynamics of the COVID-19 epidemic in Italy: effects of emergency containment measures. Proc. Natl. Acad. Sci. U.S.A. 117 (19), 10484-10491. doi:10.1073/pnas.2004978117

Giordano, G., Blanchini, F., Bruno, R., Colaneri, P., Di Filippo, A., Di Matteo, A., et al. (2020). Modelling the COVID-19 epidemic and implementation of population-wide interventions in Italy. Nat. Med. 26, 855-860. doi:10.1038/ s41591-020-0883-7

Gollwitzer, A., Martel, C., Marshall, J., Höhs, J. M., and Bargh, J. A. (2020). Connecting self-reported social distancing to real-world behavior at the individual and us state level. PsyArXiv [Preprint]. Available at: https://doi. org/10.31234/osf.io/kvnwp.

John Hopkins University (2020). COVID-19 map - Johns Hopkins coronavirus resource center. Available at: https://coronavirus.jhu.edu/map.html (Accessed July 19, 2020).

Keni, R., Alexander, A., Nayak, P. G., Mudgal, J., and Nandakumar, K. (2020). COVID-19: emergence, spread, possible treatments, and global burden. Front Public Health 8, 216. doi:10.3389/fpubh.2020.00216

Lampos, V., Moura, S., Yom-Tov, E., Cox, I. J., McKendry, R., and Edelstein, M. (2020). Tracking COVID-19 using online search. arXiv [Preprint]. Available at: https://arxiv.org/abs/2003.08086. (Accessed Jul 19, 2020).

Li, Q., Bessell, L., Xiao, X., Fan, C., Gao, X., and Mostafavi, A. (2020a). Disparate patterns of movements and visits to points of interests located in Urban hotspots across US metropolitan cities during COVID-19. arXiv preprint [Preprint]. Available at: https://arxiv.org/abs/2006.14157 (Accessed Jun 26, 2020).

Li, Q., Tang, Z., Coleman, N., and Mostafavi, A. (2020b). Detecting early-warning signals in time series of visits to points of interests to examine population response to COVID -19 pandemic. arXiv [Preprint]. Available at: https://arxiv. org/abs/2008.02905 (Accessed Aug 10, 2020).
Liu, Q.-H., Ajelli, M., Aleta, A., Merler, S., Moreno, Y., and Vespignani, A. (2018). Measurability of the epidemic reproduction number in data-driven contact networks. Proc. Natl. Acad. Sci. U.S.A. 115 (50), 12680-12685. doi:10.1073/ pnas. 1811115115

Louail, T., Lenormand, M., Cantu Ros, O. G., Picornell, M., Herranz, R., FriasMartinez, E., et al. (2014). From mobile phone data to the spatial structure of cities. Sci. Rep. 4, 5276. doi:10.1038/srep05276

$\mathrm{Lu}, \mathrm{T}$., and Reis, B. Y. (2020). Internet search patterns reveal clinical course of disease progression for COVID-19 and predict pandemic spread in 32 countries. medRxiv [Preprint]. Available at: https://doi.org/10.1101/2020.05. 01.20087858 (Accessed Sep 16, 2020).

Newman, M. E. (2002). Spread of epidemic disease on networks. Phys. Rev. E-Stat. Nonlinear Soft Matter Phys. 66 (1), 016128. doi:10.1103/PhysRevE.66.016128

Nishiura, H., and Chowell, G. (2009). The effective reproduction number as a prelude to statistical estimation of time-dependent epidemic trends-Mathematical and statistical estimation approaches in epidemiology. Dordrecht, Netherlands: Springer, 103-121.

Ramchandani, A., Fan, C., and Mostafavi, A. (2020). DeepCOVIDNet: an interpretable deep learning model for predictive surveillance of COVID-19 using heterogeneous features and their interactions. IEEE Access 8, 159915-159930. doi:10.1109/ACCESS.2020.3019989

SafeGraph (2020). Safe Graph weekly pattern Version 2. Available at: https://docs. safegraph.com/docs/weekly-patterns (Accessed Nov 4, 2020).

Tian, H., Liu, Y., Li, Y., Wu, C. H., Chen, B., Kraemer, M. U. G., et al. (2020). An investigation of transmission control measures during the first 50 days of the COVID-19 epidemic in China. Science 368 (6491), 638-642. doi:10.1126/ science.abb6105

Wang, X., Pei, T., Liu, Q., Song, C., Liu, Y., Chen, X., et al. (2020). Quantifying the time-lag effects of human mobility on the COVID-19 transmission: a multi-city study in China. IEEE Access 8, 216752-216761. doi:10.1109/ACCESS.2020. 3038995

World Health Organization (2020). WHO coronavirus disease (COVID-19) dashboard. Available at: https://covid19.who.int (Accessed Jul 19, 2020).

Wu, N., Ben, X., Green, B., Rough, K., Venkatramanan, S., Marathe, M., et al. (2020). Predicting onset of COVID-19 with mobility-augmented SEIR model. medRxiv [Preprint]. Available at: https://www.medrxiv.org/content/10.1101/ 2020.07.27.20159996v2 (Accessed July 29, 2020).

Xiong, C., Hu, S., Yang, M., Luo, W., and Zhang, L. (2020). Mobile device data reveal the dynamics in a positive relationship between human mobility and COVID-19 infections. Proc. Natl. Acad. Sci. U.S.A. 117 (44), 27087-27089. doi:10.1073/pnas.2010836117

Yabe, T., Tsubouchi, K., Fujiwara, N., Wada, T., Sekimoto, Y., and Ukkusuri, S. V. (2020). Non-compulsory measures sufficiently reduced human mobility in Japan during the COVID-19. arXiv [Preprint]. Available at: https://arxiv.org/ abs/2005.09423 (Accessed May 18, 2020).

Zhang, J., Litvinova, M., Liang, Y., Wang, Y., Wang, W., Zhao, S., et al. (2020a). Changes in contact patterns shape the dynamics of the COVID-19 outbreak in China. Science 368 (6498), 1481-1486. doi:10.1126/science.abb8001

Zhang, J., Litvinova, M., Wang, W., Wang, Y., Deng, X., Chen, X., et al. (2020b). Evolving epidemiology and transmission dynamics of coronavirus disease 2019 outside Hubei province, China: a descriptive and modelling study. Lancet Infect. Dis. 20 (7), 793-802. doi:10.1016/S1473-3099(20)30230-9

Conflict of Interest: The authors declare that the research was conducted in the absence of any commercial or financial relationships that could be construed as a potential conflict of interest.

Copyright $\odot 2021$ Gao, Fan, Yang, Lee, Li, Maron and Mostafavi. This is an openaccess article distributed under the terms of the Creative Commons Attribution License (CC BY). The use, distribution or reproduction in other forums is permitted, provided the original author(s) and the copyright owner(s) are credited and that the original publication in this journal is cited, in accordance with accepted academic practice. No use, distribution or reproduction is permitted which does not comply with these terms. 